\title{
Automatic Aerial Retrieval of a Mobile Robot Using Optical Target Tracking and Localization
}

\author{
Maximilian Laiacker, Marc Schwarzbach, Konstantin Kondak \\ German Aerospace Center DLR \\ Muenchner Str. 20 \\ 82234 Wessling, Germany \\ maximilian.laiacker@dlr.de
}

\begin{abstract}
In this paper we present a system for automatic deployment and retrieval of a mobile ground robot using a helicopter UAV. Our system allows using a mobile outdoor robot in areas that cannot be reached other than from the air and aerial measurements alone are not sufficient. For example a ground robot can perform in situ measurements and even take samples that can later be analyzed when the robot is returned by the aerial system.
\end{abstract}

We use a helicopter UAV with a rotor diameter of $1.8 \mathrm{~m}$ and a takeoff mass of $11 \mathrm{~kg}$ as a proof-of-concept platform. The UAV is equipped with our modular autopilot system. The real time control and navigation is done by the flight control computer. The target detection is done by the image processing computer connected to a downward looking camera. In addition to the autopilot payload the helicopter can carry an extra mass of around $2 \mathrm{~kg}$. The ground robot we used had a mass of $1.1 \mathrm{~kg}$ and is equipped with a GPS sensor and a communication system that is used to send its current position estimate to the UAV.

The aerial system is using a high precision hover position controller and a multi-sensor fusion module which is used for detection and precise localization of the mobile robot. It combines GPS-based localization for obtaining an initial estimation of the ground robot location and a vision-system for its accurate localization. We use a known optical marker on the ground robot for its precise localization relative to the aerial system. All control and sensor processing and fusion are performed on board of the UAV. The docking system we developed is very similar to the probe-and-drogue aerial refueling system. It is used to compensate position disturbances of the UAV during the docking maneuver. Results from multiple successful outdoor flight experiments will be presented.

\section{Table of Contents}

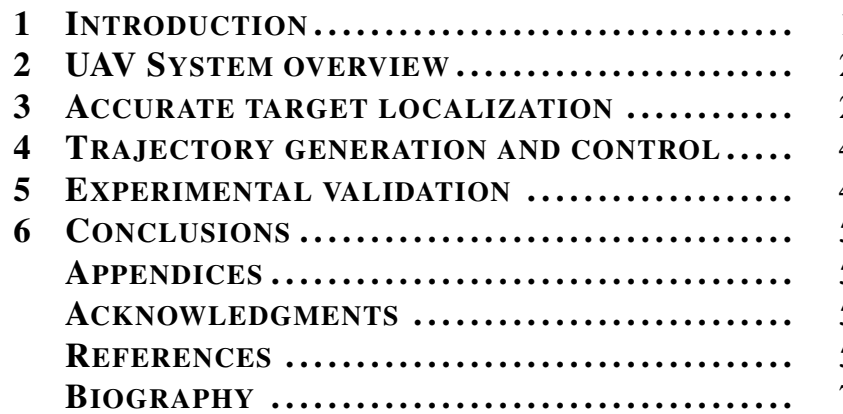

\section{INTRODUCTION}

Currently most Unmanned Aerial Vehicles (UAVs) are used for remote observation and sensing applications like search and rescue, precision farming or intelligence gathering using

978-1-4799-5380-6/15/\$31.00 @)2015 IEEE. optical and radar sensors. However, capabilities of UAVs are growing rapidly. Progress in navigation and control enables more applications. Automatic aerial refueling as presented in [1] and [2] is an example for a new UAV capability. Automatic aerial refueling requires similar techniques as those that will be presented in this paper like precision control and relative navigation. In recent publications [3], [4], [5] and [6] aerial manipulation using one or multiple quadrotors has been presented. In contrast to our approach most of these systems rely on accurate external positioning systems and the location of the objects are either known in advance or are also measured by an external positioning system. The system presented in [7] can grasp unstructured objects but needs an expert pilot to guide the helicopter manually. A slung load transport system as presented in [8] and [9] requires an operator that attaches the load to the flying system. With this system the deployment of a load in a remote location is possible but there are a lot of scenarios where the retrieval of a payload from an unaccessible location is required. One can think about a soil sample taken by a mobile robot that must be analyzed in a laboratory. In [10] and [11] we presented an aerial manipulation system using an industrial 7 degree-offreedom robot arm attached to a unmanned helicopter. This system can deploy, retrieve and even manipulate objects in a remote location. These successful experiments directed our research to several subproblems in the field of aerial manipulation which include: sensor-to-sensor calibration, sensor-to-actor calibration, object pose estimation, robot pose estimation, trajectory generation, failure detection and recovery, and precision joint control of a robot arm and flying helicopter.

This paper focuses on the estimation and calibration problems that need to be solved for reliable autonomous aerial manipulation. We decided to use a smaller and easier to operate helicopter in comparison to the one presented in [10] to validate the estimation, calibration and trajectory generation algorithms. The robot arm that compensates for positioning errors of the UAV during the manipulation was replaced by a passive compliant docking mechanism that is using an electromagnet inside a funnel mounted on a UAV. The ground object has a compliant pole mounted on the top that connects to the electromagnet. By not using an active system like a robot arm to compensate position errors during the retrieval we don't have to consider the dynamic interactions between the moving robot arm and helicopter making experimental validation of the estimation and calibration system easier. The validated estimation and calibration system presented here can then be used in a more complex aerial manipulator system. The retrieval of a mobile robot using the system is shown in Fig. 1 .

The remainder of this paper is structured as follows: Section 2 describes the docking mechanism and the sensors used for control and navigation of the UAV. The sensor calibration 


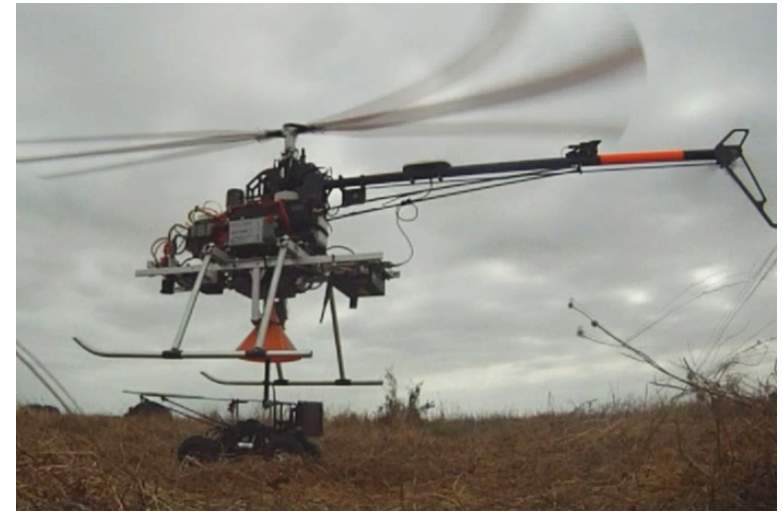

Figure 1: Automatic docking of an aerial robot to a mobile robot

and fusion used to perform the automatic aerial manipulation task is described in Section 3 The navigation and control algorithms are described in Section 4. Results of outdoor flight experiments are presented in Sect. 5. In Sect. 6 conclusions and future work are discussed.

\section{UAV SYSTEM OVERVIEW}

We use a helicopter UAV with a rotor diameter of $1.8 \mathrm{~m}$ and a takeoff mass of $11 \mathrm{~kg}$ as a proof-of-concept platform. The UAV is equipped with our modular autopilot system [12]. An RTK GPS system measures the global position of the UAV with a maximum accuracy of $\pm 1 \mathrm{~cm}$ at $25 \mathrm{~Hz}$. The inertial measurement unit (IMU) and magnetometer are used to measure acceleration and angular velocity and estimate the orientation of the UAV at $100 \mathrm{~Hz}$. The real time control and navigation is done by the flight control computer (FCC). The object detection is performed by the image processing computer connected to the downward looking camera. The camera captures gray-scale images of $1280 \times 1024$ Pixels at $30 \mathrm{~Hz}$. The camera has a field of view of about $70^{\circ}$. The FCC is connected to the image processing computer by Ethernet and to the ground station via a wireless connection. In addition to the autopilot payload the helicopter can carry an extra mass of around $2 \mathrm{~kg}$.

The docking system we developed is very similar to a "probeand-drogue" aerial refueling system. A funnel with a diameter of $16 \mathrm{~cm}$ is mounted near the center of gravity of the helicopter between the skids. An electric magnet is mounted at the top of the funnel. The magnet is turned on and off by the FCC. A flexible pole is mounted on top of the ground object near its center of gravity. This pole has a steel plate attached to the upper end which connects to the electromagnet. The combination of the funnel and the flexible pole helps to compensate position disturbances of the UAV during the docking maneuver. The magnetic docking system also has a safety feature. If the force on the docking pin exceeds about $40 N$ the payload will be released. During normal operation this force is enough to hold the payload since it has a mass of about $1.1 \mathrm{~kg}$. The UAV setup can be seen in Fig. 2.

\section{ACCURATe target localization}

The proposed docking system requires accurate estimations of the location and orientation of the target. It uses a two-

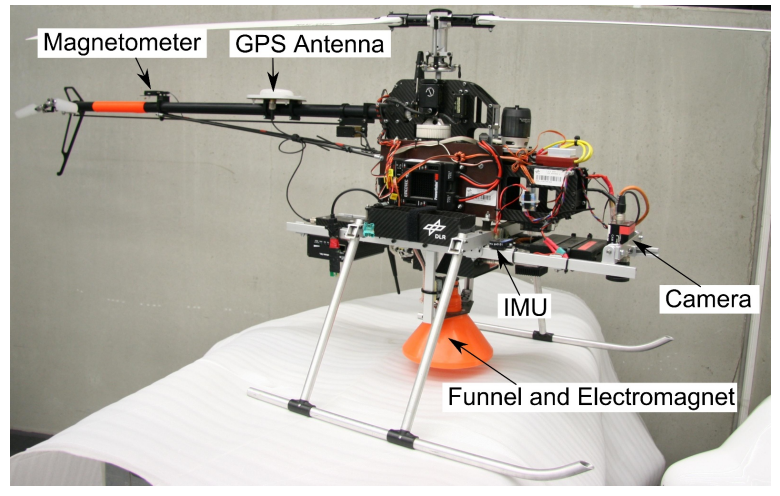

Figure 2: Helicopter system overview

stage scheme that exploits synergies resulting from integrating the measurements of multiple sensors on-board the UAV. In the first stage, the helicopter receives a position estimate from the target or uses the last known position if a stationary target is used. Next, the helicopter flies above that initial estimated position so that the target is clearly seen by the helicopter on-board camera. In the second stage, the helicopter applies vision-based methods to accurately localize the target, to which a visual marker has been attached. Both stages and the calibration of the vision method are described below.

\section{Calibration of the vision-based method}

Accurate target localization using multiple sensors requires a precise calibration. The relative pose of the sensors can be measured manually but early flight experiments had shown that the accuracy is not sufficient to perform a reliable automatic aerial manipulation. We developed an off-line calibration procedure to get a more accurate sensor-to-sensor estimate.

For this application the pose of the camera relative to the GPS antenna $\mathbf{T}_{\mathbf{C}}^{\mathbf{A}}$ needs to be known since the helicopter controller is using the GPS measurements for position control. To calculate position commands for the controller, the object localization measurements have to be transformed to the GPS antenna frame. The method used to calculate this transform is presented in the following.

The GPS position measurements $\vec{r}_{A}^{I}$ and rotation matrix $\mathbf{R}_{\mathbf{F}}^{\mathbf{I}}$ estimated by IMU measurements are combined to get the $4 \times 4$ homogeneous transformation matrix from the inertial reference frame $I$ to the GPS antenna reference frame $A$ :

$$
\mathbf{T}_{\mathbf{A}}^{\mathbf{I}}=\left[\begin{array}{cc}
\mathbf{R}_{\mathbf{F}}^{\mathbf{I}} & \vec{r}_{A}^{I} \\
0 \ldots 0 & 1
\end{array}\right]
$$

The transform that describes the pose of camera frame $C$ in the GPS antenna frame is defined as:

$$
\mathbf{T}_{\mathbf{C}}^{\mathbf{A}}\left(\mathbf{p}_{\mathbf{1} . .6}\right)=\left[\begin{array}{cc}
\mathbf{R}\left(\mathbf{p}_{\mathbf{4}}, \mathbf{p}_{\mathbf{5}}, \mathbf{p}_{\mathbf{6}}\right) & p_{1} \\
& p_{2} \\
0 \ldots 0 & p_{3} \\
0 \ldots & 1
\end{array}\right]
$$

where $\mathbf{R}\left(\mathbf{p}_{\mathbf{4}}, \mathbf{p}_{\mathbf{5}}, \mathbf{p}_{\mathbf{6}}\right)$ represents the $3 \times 3$ rotation matrix defined by the three Euler angles $p_{4}, p_{5}, p_{6}$, 
The object detection algorithm measures the position $\vec{r}_{o}^{c}$ and orientation $\mathbf{R}_{\mathbf{O}}^{\mathbf{C}}$ of the object $O$ relative to the camera frame. The position measurements may be scaled by an unknown factor, therefore the pose is defined as:

$$
\mathbf{T}_{\mathbf{O}}^{\mathbf{C}}\left(\mathbf{p}_{\mathbf{7}}\right)=\left[\begin{array}{cc}
\mathbf{R}_{\mathbf{O}}^{\mathbf{C}} & p_{7} \vec{r}_{o}^{c} \\
0 \ldots 0 & 1
\end{array}\right]
$$

Finally the pose of the object $O$ in the inertial reference frame $I$ combining all measurements is written as:

$$
\mathbf{T}_{\mathbf{O}}^{\mathbf{I}}(\tilde{\mathbf{p}})=\left[\begin{array}{cc}
\mathbf{R}_{\mathbf{O}}^{\mathbf{I}} & \vec{r}_{O}^{I} \\
0 \ldots 0 & 1
\end{array}\right]=\mathbf{T}_{\mathbf{A}}^{\mathbf{I}} \mathbf{T}_{\mathbf{C}}^{\mathbf{A}}\left(\mathbf{p}_{\mathbf{1} . .6}\right) \mathbf{T}_{\mathbf{O}}^{\mathbf{C}}\left(\mathbf{p}_{\mathbf{7}}\right)
$$

The optimal values of the parameters $\vec{p}$ can be found by solving the following nonlinear optimization problem:

$$
\underset{\vec{p} \in \mathbb{R}^{\triangleright}}{\arg \min }\left\|\sigma\left(\vec{r}_{O}^{I}(\vec{p})_{i}\right)\right\|
$$

To solve the problem the MATLAB optimization toolbox method for constrained nonlinear optimization is used. The starting values for the optimization $\vec{p}_{0}$ are chosen by measuring the position of the camera relative to the GPS antenna with a ruler and measuring the angles between the fuselage and camera. The scale parameter $p_{07}=1$ is used as initial value. The goal of the calibration is to calculate the parameters that best fit the measurements obtained during a calibration procedure. The standard deviation of object location estimation $\sigma\left(\vec{r}_{O}^{I}(p)_{i}\right)$ is chosen because the true location of the object doesn't need to be known, but it is required that the UAV is moved during the recording of calibration measurements. Without measurement noise the standard deviation for the optimum parameter $\vec{p}$ is zero given the object is stationary during the calibration.

For the calibration the UAV is moved so that the object is in the field of view of the camera and the object is detected. The values for $\mathbf{R}_{\mathbf{O}}^{\mathbf{C}}, \vec{r}_{o}^{c}$ and $\mathbf{T}_{\mathbf{A}}^{\mathbf{I}}$ are stored at each sample $i=0 . . N$ to be used in the off-line optimization. During the calibration data collection the target is rotated around all angles at least $\pm 30^{\circ}$ and moved in $x, y$ and $z$ direction. The sensor measurements are used at the sample rate of the slowest measurement. In our setup the object detection algorithm has the slowest measurement rate as it can only run as fast as the camera frame rate of $30 \mathrm{~Hz}$. Time shift between the sensor measurements and missing measurements have to be removed from the calibration data. The optimization takes less then a minute on a laptop computer using around 500 measurements.

Figure 3 shows the measured North-East coordinates of the object location in the $I$ reference frame recorded during calibration when using measured and calibrated parameters. The standard deviation is reduced from $[2.6 \mathrm{~cm}, 5.7 \mathrm{~cm}, 4.9 \mathrm{~cm}]^{T}$ to $[2.2 \mathrm{~cm}, 1.7 \mathrm{~cm}, 4.2 \mathrm{~cm}]^{T}$ when using the optimized parameters instead of the measured. These values may seem relatively small, but considering the required accuracy in the horizontal position is $7 \mathrm{~cm}$ (the funnel radius minus the radius of the docking pin) this is a significant improvement.

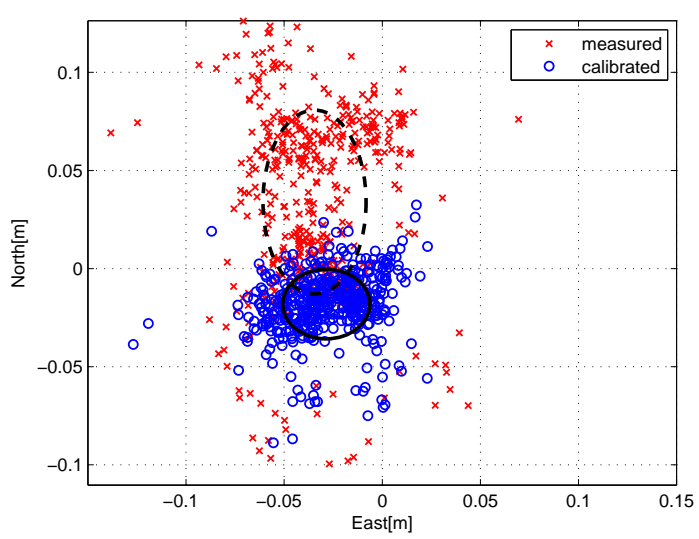

Figure 3: Measured object positions with mean and standard deviation using measured and calibrated parameters

Prior to this calibration the intrinsic camera parameters were estimated using CalLab [13]. These calibrated parameters are used in the vision based localization method to estimate the target position.

\section{Computer vision-based accurate localization}

An object localization method for aerial manipulation should provide accurate relative pose measurements. The required accuracy is higher the closer the object is to the aerial manipulator. These requirements are a perfect match for image based object detection and localization algorithms. The accuracy of most image based algorithms increases with the size of the object in the image.

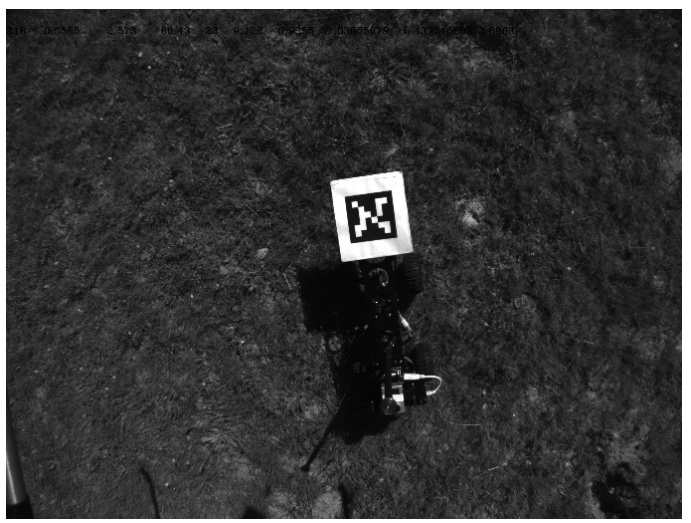

Figure 4: On board camera view of the target

We have chosen ARToolkit [14] for object detection and localization. This algorithm was chosen because it can run in real time on the on-board image processing computer. A view from the on-board camera with the target in view is shown in Fig. 4. The algorithm also has proven to be very robust against false positives and very accurate for position estimates. There is a problem that can occur when the marker is in frontal direction of the camera. In this position the orientation estimate gets unstable for the angles around the camera $x$ and $y$ axis ( $z$ axis pointing in the viewing direction) [15]. This limitation doesn't affect this application because the unstable angles are not needed for the aerial docking. Only the angle of the marker around the camera $z$ axis is 
needed to align the helicopter heading to the object.

The object detection algorithm provides a relative position and orientation estimate $\mathbf{T}_{\mathbf{O}}^{\mathbf{C}}$ of the target relative to the camera. To use this measurements for the helicopter position controller it needs to be transformed into a relative position in the inertial frame $\Delta \mathbf{T}_{\mathbf{O}}^{\mathbf{I}}$ which can be calculated as follows:

$$
\Delta \mathbf{T}_{\mathbf{O}}^{\mathbf{I}}=\left[\begin{array}{cc}
\mathbf{R}_{\mathbf{F}}^{\mathbf{I}} & \mathbf{0} \\
\mathbf{0} & 1
\end{array}\right]\left[\begin{array}{cc}
\mathbf{R}_{\mathbf{C}}^{\mathbf{A}} & \mathbf{0} \\
\mathbf{0} & 1
\end{array}\right] \mathbf{T}_{\mathbf{O}}^{\mathbf{C}}
$$

where $\mathbf{R}_{\mathbf{C}}^{\mathbf{A}}$ equals $\mathbf{R}\left(\mathbf{p}_{\mathbf{4}}, \mathbf{p}_{\mathbf{5}}, \mathbf{p}_{\mathbf{6}}\right)$ calculated during the GPS camera calibration. From $\Delta \mathbf{T}_{\mathbf{O}}^{\mathbf{I}}$ only the $x, y, z$ and $y a w$ values are used to update the target position for the helicopter controller.

\section{TRAJECTORY GENERATION AND CONTROL}

Several steps are required to fulfill the task of automatic retrieval of objects on the ground.

A rough estimate of the position of the target is needed. In our case it is provided by the target over a communication link or by the last know position. The helicopter is commanded to a safe altitude above the target using standard waypoint guidance. After reaching the waypoint, the helicopter lowers its altitude to allow the vision system to pick up the object.

When object tracking is confirmed, the altitude is lowered again. At the same time, the helicopter heading is aligned to the object for docking. The desired heading is also provided by the object localization. By closely integrating visual measurements, which are very accurate at close distance, the relative position is determined at high frequency and high accuracy. After a short time at an altitude where the magnet should capture the object, the helicopter flies up to a certain altitude. While doing that, a check is performed if contact was successful.

Should large deviations in absolute position occur close to the ground, the retrieval attempt is stopped and the altitude set to a safe value to start again.

In case the object retrieval was successful, the helicopter climbs to a safe altitude and flies to a desired waypoint for further tasks.

A high performance position control of the helicopter is required to ensure the reliability of the docking. In a hovering state close to the ground, before and during the docking, the helicopters attitude and position are influenced by air flow redistribution between the helicopters main rotor and the ground. These air movements in ground effect are difficult to model and to predict. Therefore the corresponding influence of the helicopter should be rejected by the helicopter controller. In the presented system a high performance position controller composed of two loops was applied. In the inner loop the attitude and in the outer loop the position are controlled. In both loops linear controllers with inverse dynamical blocks for linearization and decoupling are used [16]. To ensure a high performance in position control both loops are tuned to their limits accounting for the interaction between the loops. For that the coefficients of the linear controllers are calculated using pole placement method and a linear model of the whole system. The tuning parameters for flight experiments are the position of the poles and overall gain factor which considers uncertainties in the modeling of the torque generation on the main rotor. Moving the poles to one position, we reduce the number of tuning parameters to two. This makes the experimental tuning feasible with a moderate effort and ensures a high performance of the resulting controller. The guidance, navigation and control sceme used is shown in Figure 5 .

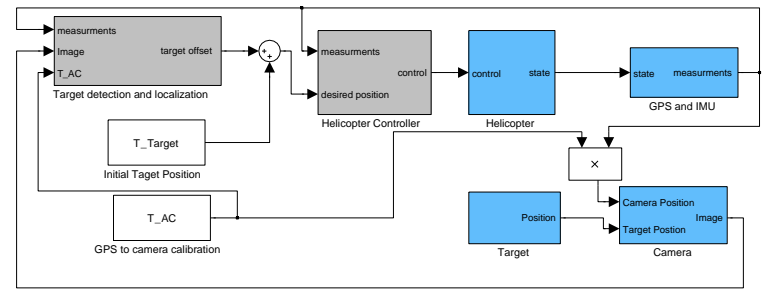

Figure 5: Diagram of the vision aided helicopter control for aerial docking

\section{EXPERIMENTAL VALIDATION}

The docking experiments consisted in collecting, transporting and deploying a ground robot. The joint aerial and ground system was used for exploration of inaccessible areas.

The system was validated in several flight experiments. The experiments where performed entirely under autopilot control. A marker with a size of $9 \times 9 \mathrm{~cm}^{2}$ was placed on top of the ground robot. The flight experiments have shown that the marker is reliably detected up to distance of about $4 \mathrm{~m}$ with the camera used. The marker size was chosen based on the available space on the mobile robot and the field of view of the camera during the docking. If the boarders of the marker are not visible in the image the detection doesn't work. With the chosen size the marker fills about a fourth of the image when the UAV is in the docking position. This allows detection of the marker even if the UAV has to perform orientation and position corrections during the final docking phase.

The experiment begins with the helicopter on a starting platform and the safety pilot enabling the autopilot. The helicopter performs an automatic take-off. After the take-off the helicopter estimates the location of the ground robot and is commanded to fly above that position. When the position above the ground robot is reached the algorithm described in Sec. 4 is activated and the robot is picked up from the ground and then transported to a position commanded by the operator and dropped on the ground from low altitude. After dropping the mobile robot an automatic landing is executed. The data recorded during one experiment is presented in the following. The distance in the North-East (NE) plane relative to the docking pin measured by the object localization during four docking attempts is shown in Fig. 7. The fourth attempt was successful. Three successive still images taken from a video of the successful docking are shown in Fig. 6.

The docking sequence starts when the robot is detected while hovering over the position provided by the mobile robot. The helicopter is then approaching the robot from above and also aligning the heading angle to that of the mobile robot. While reducing the altitude a successful docking can only be made when the lower side of the funnel is lowered below the docking pin and at the same time the NE distance is smaller then the funnel radius minus the radius of the docking pin. This can clearly be seen during the successful 4 . attempt 

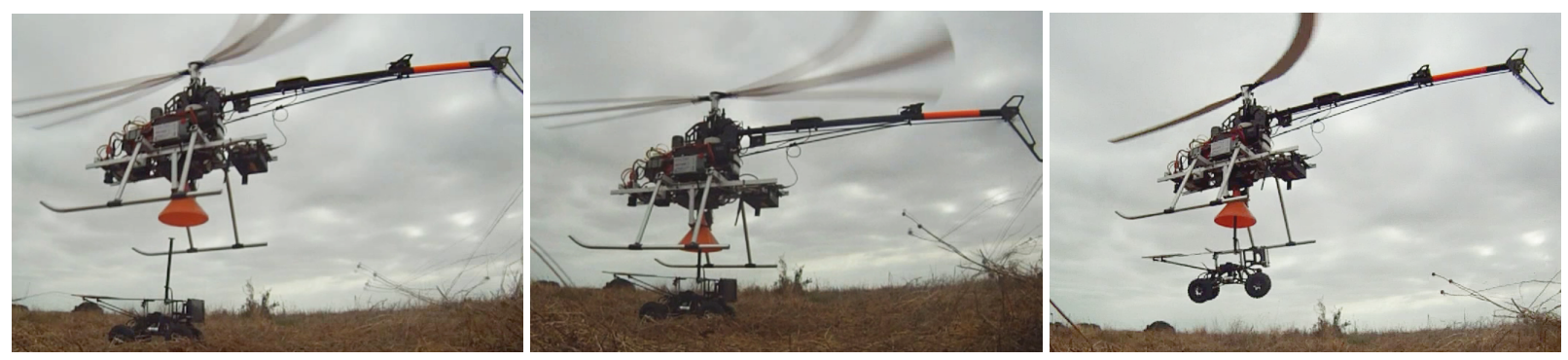

Figure 6: Sequence of the UAV helicopter automatically docking and lifting a mobile robot

in Figure 7 and four successful docking experiments from a second flight experiment are shown in Figure 8 The moment when the docking pin is below the end of the funnel is marked by a vertical blue line. The black areas highlight the time the NE distance is smaller then the funnel radius. During the fourth and successful attempt in Fig. 7 the NE distance is below $4 \mathrm{~cm}$ when the pin enters the funnel at about $166.2 \mathrm{~s}$. After the pin enters the funnel the NE distance gets bigger then the funnel radius but now the funnel can guide the pin towards the electromagnet and a successful docking can be performed. During the second flight experiment shown in Fig. 8 all four docking maneuvers where successful. When a successful docking is detected the helicopter will fly up vertically lifting up the mobile robot. A failed docking is detected when the event marked by the blue line happens outside a black area which happens during attempt 1 to 3 in Fig. 7. When a failed docking attempt is detected the helicopter will fly up until the docking pin is below the funnel and then start again.

These experiments show that the estimation and calibration algorithm described in this paper allows a helicopter to perform automatic aerial docking.

\section{Conclusions}

We presented a multi-sensor estimation and calibration system by combining visual object detection, IMU and GPS measurements that is able to guide a UAV for precise approach and retrieval of a mobile robot from the ground. Using the proposed system the position of the helicopter relative to the target can be measured precisely. The flight experiments have shown that it also allows automatic detecting if the docking is successful or should be aborted.

Future work will focus on improving the reliability and autonomy of aerial manipulation systems. This will be achieved by improving the modeling and control of the aerial system. Improvement can also be made by using more advanced object detection algorithms and including image based navigation to provide position and velocity information during GPS outages.

\section{APPENDICES}

\section{ACKNOWLEDGMENTS}

This work has been partially supported by PLANET, Platform for the Deployment and Operation of Heterogeneous Networked Cooperating Objects, funded by the European Commission under FP7 with contract FP7-2009-5-257649.

\section{REFERENCES}

[1] M. Mammarella, G. Campa, M. Napolitano, M. Fravolini, Y. Gu, and M. Perhinschi, "Machine vision/gps integration using ekf for the uav aerial refueling problem," Systems, Man, and Cybernetics, Part C: Applications and Reviews, IEEE Transactions on, vol. 38, no. 6, pp. 791-801, Nov 2008.

[2] Z. Mahboubi, Z. Kolter, T. Wang, G. Bower, and A. Y. $\mathrm{Ng}$, "Camera based localization for autonomous uav formation flight," in Proceedings of the AIAA@ Infotech Conference, 2011.

[3] D. Mellinger, Q. Lindsey, M. Shomin, and V. Kumar, "Design, modeling, estimation and control for aerial grasping and manipulation," in IEEE/RSJ Intl. Conf. on Intelligent Robots and Systems, IROS2011, Sept 2011, pp. 2668-2673.

[4] D. Mellinger, M. Shomin, N. Michael, and V. Kumar, "Cooperative grasping and transport using multiple quadrotors," in Distributed Autonomous Robotic Systems, ser. Springer Tracts in Advanced Robotics, A. Martinoli, F. Mondada, N. Correll, G. Mermoud, M. Egerstedt, M. A. Hsieh, L. E. Parker, and K. Sty, Eds. Springer Berlin Heidelberg, 2013, vol. 83, pp. 545-558. [Online]. Available: http://dx.doi.org/10.1007/978-3-642-32723-0_39

[5] M. Orsag, C. Korpela, and P. Oh, "Modeling and control of mm-uav: Mobile manipulating unmanned aerial vehicle," Journal of Intelligent \& Robotic Systems, vol. 69, no. 1-4, pp. 227-240, 2013.

[6] J. Willmann, F. Augugliaro, T. Cadalbert, R. D'Andrea, F. Gramazio, and M. Kohler, "Aerial robotic construction towards a new field of architectural research," International Journal of Architectural Computing, vol. 10, no. 3, pp. 439-460, 2012.

[7] P. E. I. Pounds, D. Bersak, and A. Dollar, "Practical aerial grasping of unstructured objects," in Technologies for Practical Robot Applications (TePRA), 2011 IEEE Conference on, April 2011, pp. 99-104.

[8] M. Bernard and K. Kondak, "Generic slung load transportation system using small size helicopters," in ICRA. IEEE, 2009, pp. 3258-3264.

[9] M. Bernard, K. Kondak, I. Maza, and A. Ollero, "Autonomous transportation and deployment with aerial robots for search and rescue missions," J. Field Robotics, vol. 28, no. 6, pp. 914-931, 2011.

[10] F. Huber, K. Kondak, K. Krieger, D. Sommer, M. Schwarzbach, M. Laiacker, I. Kossyk, S. Parusel, S. Haddadin, and A. Albu-Schaffer, "First analysis and experiments in aerial manipulation using fully actuated redundant robot arm," in IEEE/RSJ Intl. Conf. on Intel- 

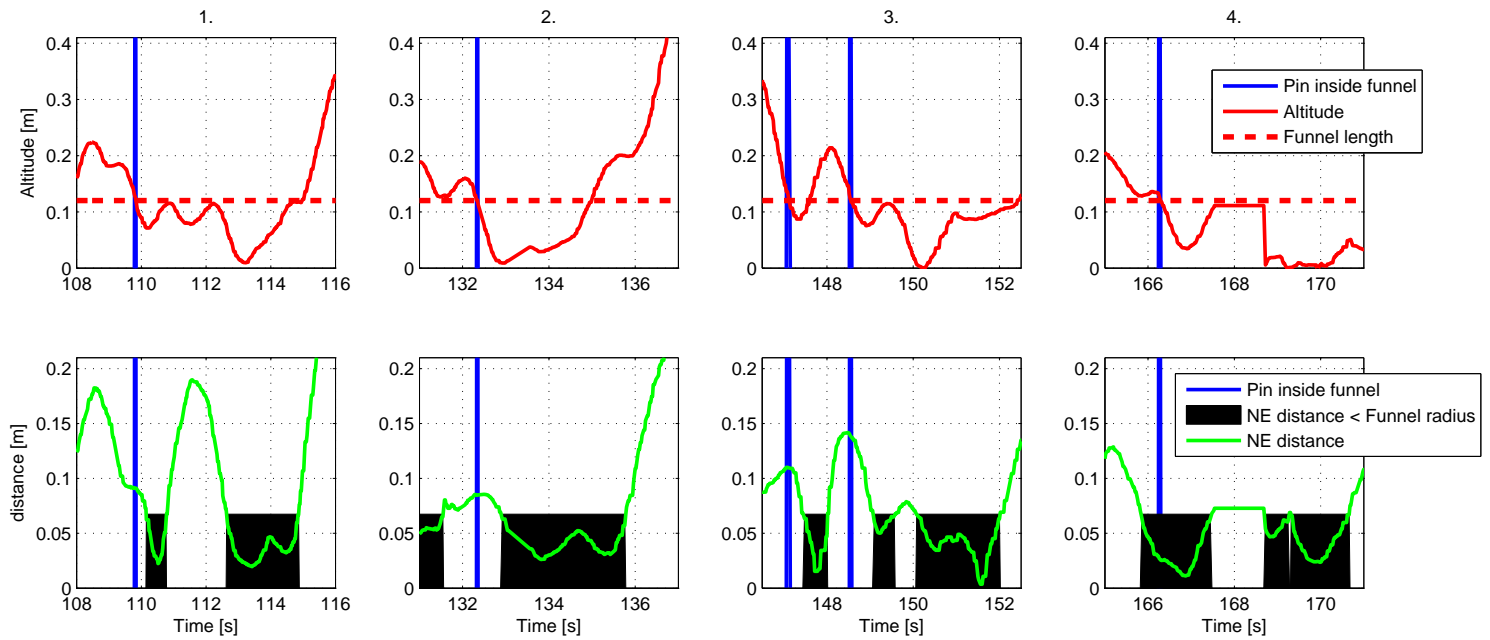

Figure 7: Automatic docking flight experiments
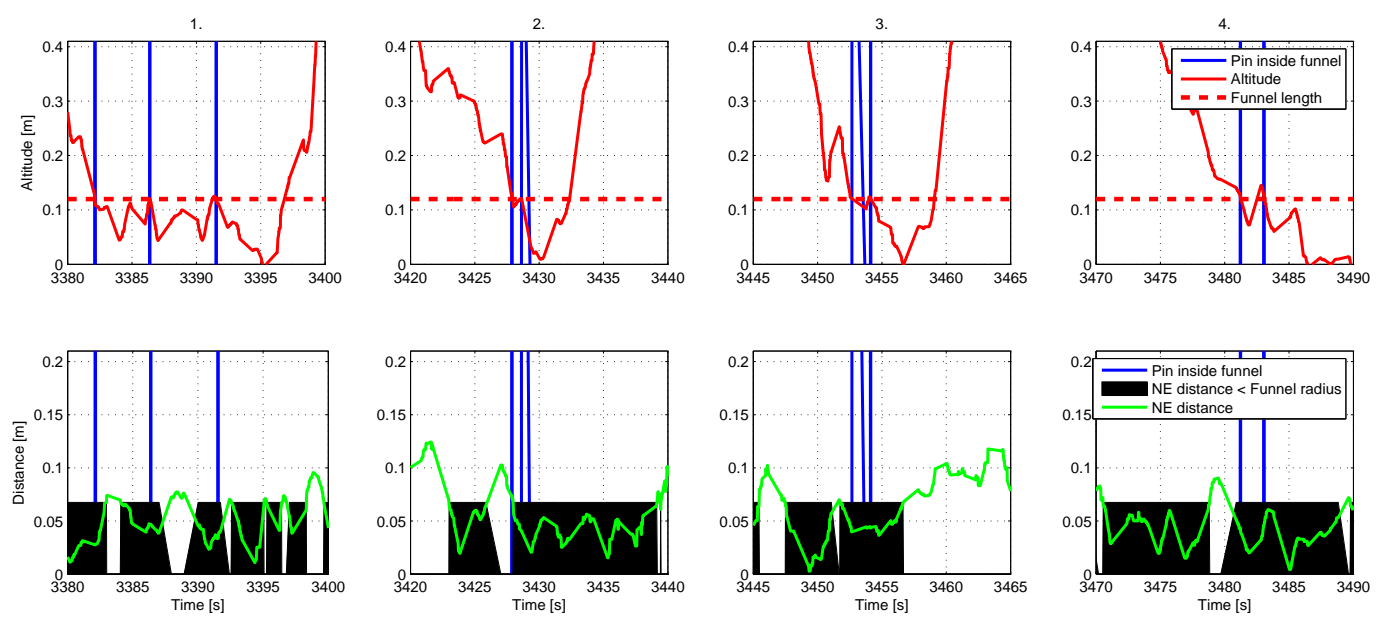

Figure 8: Second automatic docking flight experiments

ligent Robots and Systems, IROS2013, 2013, pp. 34523457.

[11] K. Kondak, K. Krieger, A. Albu-Schaeffer, M. Schwarzbach, M. Laiacker, I. Maza, A. RodriguezCastano, and A. Ollero, "Closed-loop behavior of an autonomous helicopter equipped with a robotic arm for aerial manipulation tasks," Int J Adv Robotic Sy, vol. 10 , no. $145,2013$.

[12] M. Laiacker, A. Klöckner, K. Kondak, M. Schwarzbach, G. Looye, and D. S. I. Kossyk, "Modular scalable system for operation and testing of uavs," in American Control Conference, 2013.

[13] K. H. Strobl and G. Hirzinger, "More accurate pinhole camera calibration with imperfect planar target," in IEEE Intl. Conf. on Computer Vision Workshops (ICCV Workshops), 2011, pp. 1068-1075.

[14] D. Wagner and D. Schmalstieg, "Artoolkitplus for pose tracking on mobile devices," in Proceedings of 12th Computer Vision Winter Workshop (CVWW'07), 2007, pp. 139-146.
[15] H. Tanaka, Y. Sumi, and Y. Matsumoto, "A highaccuracy visual marker based on a microlens array," in IEEE/RSJ Intl. Conf. on Intelligent Robots and Systems, IROS2012, 2012, pp. 4192-4197.

[16] K. Kondak, M. Bernard, N. Meyer, and G. Hommel, "Autonomously flying vtol-robots: Modeling and control," in ICRA. IEEE, 2007, pp. 736-741. 


\section{BIOGRAPHY}
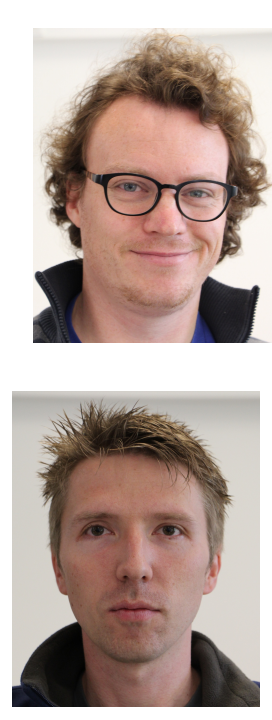

Maximilian Laiacker received his diploma in computer science from $T U$ Berlin in 2009. He is currently a member of the Flying Robots research group at the Robotics and Mechatronics Center of the German Aerospace Center DLR. His main research interests are vision based state estimation, collision avoidance and sensor fusion for flying robots.

Marc Schwarzbach received his diploma in aerospace engineering from University of Stuttgart in 2005 and a Ph.D. in engineering in 2012. He is currently a member of the Flying Robots research group at the Robotics and Mechatronics Center of the German Aerospace Center DLR. His current research is focused on flying robotics both in the high altitude and close to ground domain.

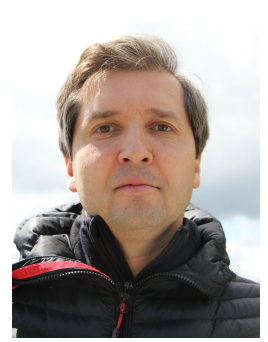

Konstantin Kondak received his diploma in Computer Engineering, his doctoral and postdoctoral degree from the $T U$ Berlin in 1999, 2001 and 2007 respectively. From 06.2008 to 10.2009, he holds the position of the representative full professor at the Real-Time Systems and Robotics Group at the TU Berlin. Since 07.2008, he was the head of the laboratory for exoskeleton robots and since 06.2006 the head of the laboratory for autonomous flying robots. Since 10.2009 he is the head of key research area flying robots at DLRs institute of Robotics and Mechatronics. 